\title{
A heterogeneous "resting" pool of synaptic vesicles that is dynamically interchanged across boutons in mammalian CNS synapses
}

\author{
Tomas Fernandez-Alfonso ${ }^{1,2}$ and Timothy A. Ryan ${ }^{1, *}$ \\ ${ }^{1}$ Department of Biochemistry, Weill Medical College of Cornell University, 1300 York Ave, \\ New York, NY 10021, USA ( ${ }^{*}$ author for correspondence; e-mail: taryan@med.cornell.edu) \\ ${ }^{2}$ Present address: Department of Neuroscience, Physiology and Pharmacology, University College \\ London, Gower Street, London NW5 1LY, UK
}

Received 2 April 2008; Revised 10 August 2008; Accepted 20 August 2008

Published online 22 October 2008

(c) The Author(s) 2008. This article is published with open access at Springerlink.com

\begin{abstract}
Using pHluorin-tagged synaptic vesicle proteins we have examined the partitioning of these probes into recycling and nonrecycling pools at hippocampal nerve terminals in cell culture. Our studies show that for three of the major synaptic vesicle components, vGlut-1, VAMP-2, and Synaptotagmin I, 50$60 \%$ of the tagged protein appears in a recycling pool that responds readily to sustained action potential stimulation by mobilizing and fusing with the plasma membrane, while the remainder is targeted to a nonrecycling, acidic compartment. The fraction of recycling and nonrecycling (or resting) pools varied significantly across boutons within an individual axon, from $100 \%$ resting (silent) to $100 \%$ recycling. Single-bouton bleaching studies show that recycling and resting pools are dynamic and exchange between synaptic boutons. The quantitative parameters that can be extracted with the approaches outlined here should help elucidate the potential functional role of the resting vesicle pool.
\end{abstract}

\section{Introduction}

At nerve terminals, synaptic vesicles recycle to sustain neurotransmitter release during repetitive action potential (AP) firing. The size of the pool of vesicles that recycle and the kinetics of each of the recycling steps all play important roles in determining the efficacy of synaptic function during repetitive stimulation (Betz and Angleson, 1998; von Gersdorff and Matthews, 1999; Fernandez-Alfonso and Ryan, 2006). Traditionally, vesicle pools at synaptic terminals have been defined based on kinetic criteria related to their depletion rates (Elmqvist and Quastel, 1965; von Gersdorff and Matthews, 1997; Dobrunz and Stevens, 1997; Wu and Betz, 1998). The readily releasable pool is thought to correspond to vesicles in immediate apposition to the active zone (so-called docked vesicles) that undergo exocytosis during a small number of AP stimuli. Beyond the active zone, presynaptic terminals have clusters of dozens to hundreds of synaptic vesicles that are recruited for exocytosis during prolonged stimulation and are referred to as the reserve pool (Stevens and Tsujimoto, 1995; Pieribone et al., 1995; Neher, 1998). The entire recycling vesicle pool is comprised of both the readily releasable and reserve pool of vesicles. FM dye labeling of synaptic vesicles in hippocampal nerve terminals show that the entire pool of recycling vesicles can be turned over with a few hundred stimuli, i.e., the amount of FM loaded saturates after a few hundred stimuli, and a similar number of stimuli causes $>90 \%$ of the dye to be released (Ryan and Smith, 1995; 
Ryan et al., 1996). However, the number of vesicles loaded with such protocols appears significantly lower than those independently observed in electron micrographs (Murthy et al., 1997; Murthy and Stevens, 1999). Additionally, ultrastructural localization of FM dyes, following photoconversion to an electron dense product, shows that only a fraction of the vesicles appear to have acquired dye even after loading under maximal stimulation (Harata et al., 2001). Recently, using synapto-pHluorin to label synaptic vesicles, Li et al. (2005) suggested that some vesicles existed in a resting pool that would not fuse with the plasma membrane upon strong stimulation; however, a detailed quantification of this pool or its dynamics across boutons and during long time scales was not provided. The simplest interpretation of these data is that a portion of the vesicle pool fails to recycle (Murthy et al., 1997; Murthy and Stevens, 1999; Sudhof, 2000). Similar observations have been made in the Calyx of Held, a giant auditory brain stem synapse, where exhaustive FM labeling resulted in only a minor fraction of ultrastructurally identifiable synaptic vesicle labeling (de Lange et al., 2003). Here, we have sought to examine the characteristics of both recycling and resting vesicle pools in a quantitative fashion in live cells. We made use of pHluorin-tagged synaptic vesicle proteins and the $\mathrm{pH}$ sensitivity of these probes to determine when and if they can be mobilized to fuse with the plasma membrane at synaptic terminals.

pHluorin is a modified form of GFP whose $\mathrm{pH}$ sensitivity has been shifted relative to that of EGFP by one log unit such that the $p K_{\mathrm{a}}$ is $\sim 7.1$ (Sankaranarayanan et al., 2000). Upon binding the protons the fluorescence is completely quenched, and therefore the fluorescence reflects the fraction of the time the fluorescent protein is in the protonbound state. pHluorins can be targeted to the lumen of synaptic vesicles by fusion to one of the 10 identified synaptic vesicle proteins with membrane spanning domains (Takamori et al., 2006). When expressed in neurons these proteins provide an useful readout of synaptic exocytosis and synaptic vesicle recycling. To date, four of the most abundant synaptic vesicle proteins, VAMP-2 (Miesenbock et al., 1998), vGlut-1 (Voglmaier et al., 2006), Synaptotagmin I (Fernandez-Alfonso et al., 2006), and Synaptophysin (Granseth et al., 2006) have been successfully used for examining cycles of exo-endocytosis at nerve terminals with pHluorin tagging.

During synaptic vesicle recycling, the lumen of the vesicle is reacidified by virtue of the action of a V-type ATPase that develops a pH gradient and the proton motive force providing the energy to drive neurotransmitter uptake. When an inhibitor of this proton pump is applied to synapses during repetitive stimulation, vesicles continue to recycle; however, they fail to reacidify and remain alkaline after endocytosis (termed alkaline trapping, Sankaranarayanan and Ryan, 2001). Resting vesicles remain acidic and can be quantified by use of $\mathrm{NH}_{4} \mathrm{Cl}$ that can rapidly alkanalize internal organelles. Comparison of the steady-state level of fluorescence achieved with pHluorin-tagged vesicle proteins during prolonged AP stimulation and alkaline trapping, with that achieved by direct alkalization of the entire vesicle pool provides a real time estimate of the fraction of the total pool of these vesicle proteins that remain acidic and hence fails to recycle.

Using these approaches, we show that a variable fraction of the total pool of the synaptic vesicle proteins VAMP-2, vGlut-1, and Synaptotagmin I do not participate in recycling during repetitive stimulation, but are dynamically exchanged between synapses.

\section{Results}

\section{Measuring the fraction of the total pool of vesicles that participates in recycling}

The main goal of this work was to quantify the fraction of the synaptic vesicle (SV) pool located at presynaptic terminals that participates in recycling during prolonged AP firing. We developed a protocol using pHluorin as an optical tracer to visualize and quantify both the total SV pool and the fraction of it that can be engaged to recycle. We have explicitly assumed that synaptic vesicles are the only $\mathrm{spH}$-containing organelles in synaptic terminals (see Discussion). Figure 1a shows confocal images of a rat hippocampal neuron in culture transfected with $\mathrm{spH}$, depicting GFP fluorescence intensity in a pseudo-color scale. The fluorescence of this $\mathrm{pH}$-sensitive GFP fused to the lumenal aspect of VAMP-2 is largely quenched at the resting lumenal $\mathrm{pH}$ (5.5), and undergoes an 

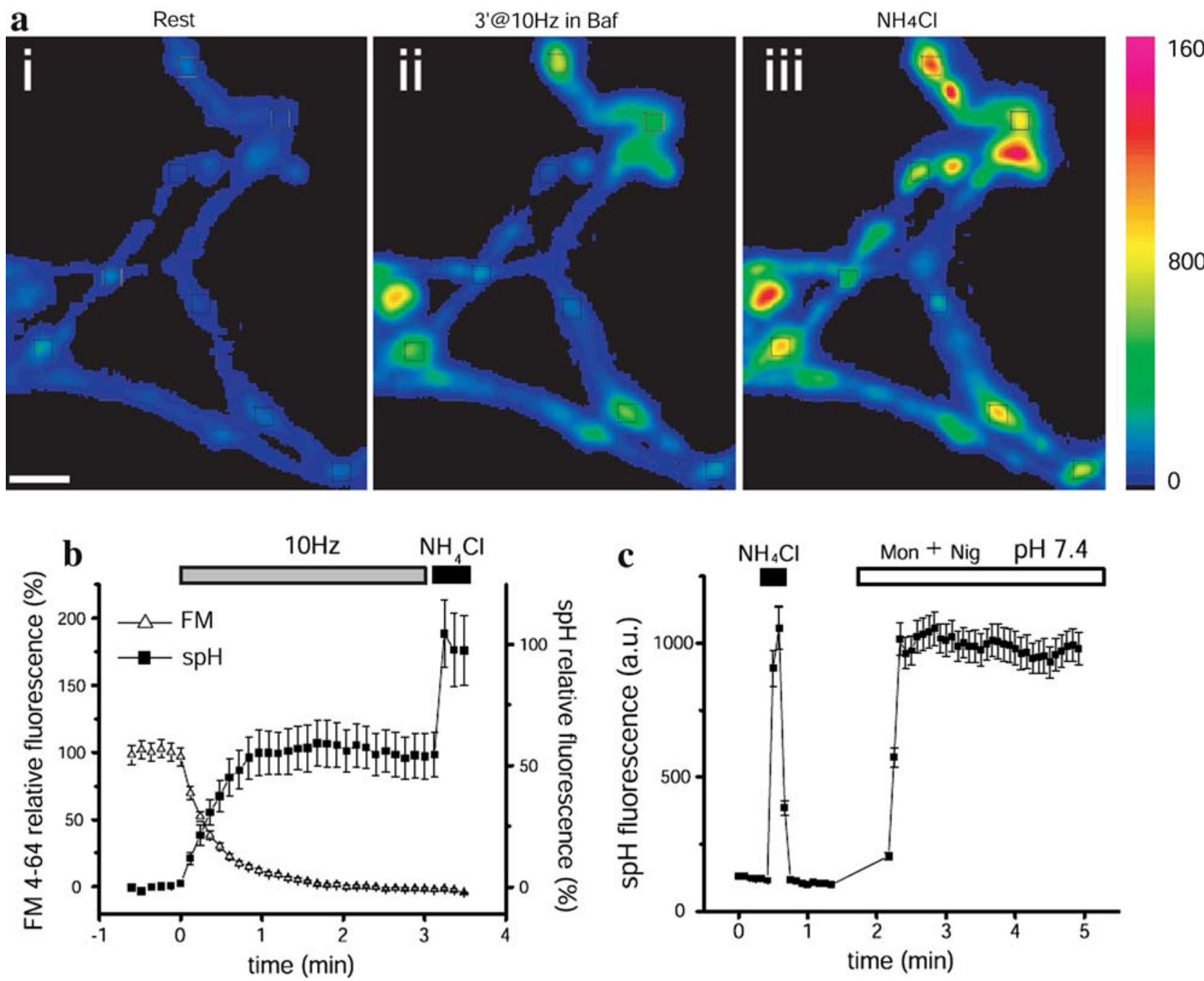

Fig. 1. Quantitative estimates of recycling and resting vesicle pool fractions in live cells. (a) Pseudo-color images of a representative axonal branch from a spH-transfected hippocampal neuron, during rest (i), after field stimulation with 1800 APs in the presence of $0.5 \mu \mathrm{M}$ Bafilomycin (Baf) (ii), and during a subsequent application of $\mathrm{NH}_{4} \mathrm{Cl}(50 \mathrm{mM}, \mathrm{pH} 7.4)$ to equilibrate synaptic-vesicle lumen pH to 7.4 (iii). Black boxes represent examples of areas selected for analysis. Only a subset is shown for clarity. Scale bar, $3 \mu \mathrm{m}$. Vertical pseudo-color scale shows spH fluorescence intensity values (arbitrary units, a.u.). (b) Representative experiment showing the protocol used to visualize the fraction of the total pool of synaptic vesicles participating in recycling. (Solid squares) Average spH fluorescence response of one spHtransfected neuron ( $n=49$ boutons) to $1800 \mathrm{APs}(10 \mathrm{~Hz}$, gray bar) normalized to the fluorescence level obtained during a subsequent $\mathrm{NH}_{4} \mathrm{Cl}$ exposure (black bar). Baf $(0.5 \mu \mathrm{M})$ was present throughout the experiment to prevent reacidification of vesicles after endocytosis (alkaline trapping); therefore any increase in $\mathrm{spH}$ fluorescence during stimulation reflects new vesicles undergoing exocytosis. The increase in fluorescence reached a steady-state level after $\sim 600 \mathrm{AP}$, indicating the time required to turn over the recycling pool of vesicles. $\mathrm{NH}_{4} \mathrm{Cl}$ application produced a further increase in fluorescence, reflecting the equilibration of resting vesicles to $\mathrm{pH}$ 7.4. FM 4-64 unloading (open triangles) was simultaneously recorded in the same set of boutons, which were preloaded with dye in a previous round of stimulation. The time to reach $50 \%$ recycling pool turnover was identical based on either spH or FM 4-64 responses $(\sim 180 \mathrm{AP})$. (c) $\mathrm{NH}_{4} \mathrm{Cl}$ effectively "unmasks" the total pool of synaptic vesicles present at axon terminals. The spH fluorescence level reached with $\mathrm{NH}_{4} \mathrm{Cl}(\mathrm{black}$ bar) is the same as that reached when the $\mathrm{pH}$ of the vesicles was equilibrated to the extracellular $\mathrm{pH}(7.4)$ by applying $40 \mu \mathrm{M}$ Monensin and $10 \mu \mathrm{M}$ Nigericin (Mon + Nig, white bar), which allow neutral exchange of cations for protons across cell membranes. 
20-fold increase in its fluorescence when the $\mathrm{pH}$ shifts to 7.4 upon exocytosis (Miesenbock et al., 1998; Sankaranarayanan et al., 2000). The fluorescence observed at rest (panel $i$ in Fig. 1a) largely reflects VAMP-2 on the plasma membrane surface. Superfusion with $\mathrm{NH}_{4} \mathrm{Cl}(50 \mathrm{mM})$ equilibrates the SV lumen to a $\mathrm{pH}$ of 7.4 (Miesenbock et al., 1998), thus unmasking clusters of vesicles located in axon terminals and allowing the visualization of individual boutons as puncta of fluorescence (panel iii). These puncta were chosen as regions of analysis in subsequent studies (square boxes in Fig. 1a, see section "Experimental procedures"). Synaptic vesicles are normally re-acidified after endocytosis by a V-type ATPase whose action can be blocked by the reversible cellpermeant inhibitor bafilomycin (baf). Measurements of $\mathrm{spH}$ fluorescence during AP stimulation in baf provide a direct measure of exocytosis, independent of any endocytic event, since vesicles become trapped in the alkaline state during recycling (alkaline trapping). Bafilomycin does not significantly perturb exocytosis or endocytosis in this system (Cousin and Nicholls, 1997; Sankaranarayanan and Ryan, 2001; Zhou et al., 2000; Li et al., 2005). To visualize the pool of vesicles that can be engaged to recycle, neurons were field stimulated with $1800 \mathrm{APs}$ at $10 \mathrm{~Hz}$ (which far exceeds the stimulus required to turn over the entire recycling pool of SVs, see below) in the presence of baf (panel ii). The size of the vesicle pool that participates in recycling can be estimated for each bouton by subtracting the fluorescence during rest from that obtained after the stimulus $\left(\Delta F_{1800 \mathrm{AP}}\right.$, i.e., panel ii - panel i). A similar subtraction can be done to the fluorescence in $\mathrm{NH}_{4} \mathrm{Cl}$ to get the size of the total vesicle pool $\left(\Delta F_{\mathrm{NH}_{4} \mathrm{Cl}}\right.$, i.e., panel iii - panel i). Finally, the fraction of the total pool of vesicles recycling can be calculated for each bouton as $\Delta F_{1800 \mathrm{AP}} / \Delta F_{\mathrm{NH}_{4} \mathrm{Cl}}$. Figure $1 \mathrm{~b}$ shows the $\mathrm{spH}$ fluorescence increase during stimulation in the presence of baf (alkaline trapping) and a subsequent application of $\mathrm{NH}_{4} \mathrm{Cl}$, plotted as a function of time. A steady-state level is reached after $\sim 600 \mathrm{APs}$, reflecting the stimulation required to turn over the entire recycling pool (Fig. 1b, solid squares). This complete turnover was confirmed by simultaneously measuring FM 4-64 destaining from the same set of boutons (Fig. 1b, open triangles), which were preloaded with the dye in a previous round of stimulation. FM 4-64 destaining kinetics agreed quantitatively very well with the kinetics of alkaline-trapped $\mathrm{spH}$ fluorescence increase (time to half FM signal decay $=18 \mathrm{~s}$, time to half $\mathrm{spH}$ signal increase $=20 \mathrm{~s}$ ), further supporting the idea that this methodology provides a robust measure of AP-driven synaptic vesicle pool turnover. In general, this steady-state level in $\mathrm{spH}$ fluorescence was no different if neurons were stimulated at a frequency of $20 \mathrm{~Hz}$ and it was reached within 6001200 APs, in agreement with previous reports (Ryan and Smith, 1995). To confirm that $\mathrm{NH}_{4} \mathrm{Cl}$ (50 mM, pH 7.4) effectively equilibrates the lumen of synaptic vesicles with the extracellular $\mathrm{pH}$, the peak fluorescence level reached in $\mathrm{NH}_{4} \mathrm{Cl}$ was compared with that reached when a combination of ionophores that allow free exchange of cations for protons across cell membranes was applied. Figure $1 \mathrm{c}$ shows such an experiment. Monensin $(40 \mu \mathrm{M})$ and nigericin $(10 \mu \mathrm{M})$ were perfused in a $\mathrm{Ca}^{2+}$ and $\mathrm{Na}^{+}$-free (replaced with $\mathrm{K}^{+}$) saline to prevent vesicle fusion and allow $\mathrm{pH}$ equilibration across all membranous compartments (Lee and Epel, 1983; Lemasters et al., 1995). spH reached the same fluorescence level in both conditions (peak mon + nig/peak $\mathrm{NH}_{4} \mathrm{Cl}=0.96 \pm 0.1$, Mean \pm SD of 6 independent experiments), indicating that $\mathrm{pH}$ inside SV-lumen is effectively equilibrated with the extracellular $\mathrm{pH}$ during $\mathrm{NH}_{4} \mathrm{Cl}$ exposure. Taken together, these experiments indicate that $\mathrm{spH}$ fluorescence values reached during prolonged stimulation in baf, as compared to that reached under $\mathrm{NH}_{4} \mathrm{Cl}$, provides a good estimate of the fraction of the total pool of synaptic vesicles that can be engaged in recycling as well as the fraction that remains acidic and hence refractory to recycling.

\section{The fraction of recycling vesicles varies from synapse to synapse and neuron to neuron} The distribution of recycling vesicle fractions in a population of 47 boutons arising from an individual neuron is presented in Fig. 2. For each bouton, the $\mathrm{spH} \Delta \mathrm{F}$ during both alkaline trapping and $\mathrm{NH}_{4} \mathrm{Cl}$ application is depicted in Fig. 2a. Displaying the fraction recycling as a function of the entire vesicle pool shows that that terminals with larger vesicle pools do not necessarily have larger recycling fractions (Fig. 2b, but see also below and Fig. 3d). Figure $2 c$ shows the frequency distribution of the fraction recycling for the 47 boutons analyzed. The percentage of vesicles recycling for this neuron varied from $0 \%$ to $100 \%$ for different boutons, with a mean of $54 \%$ with a standard deviation of $27 \%$. 

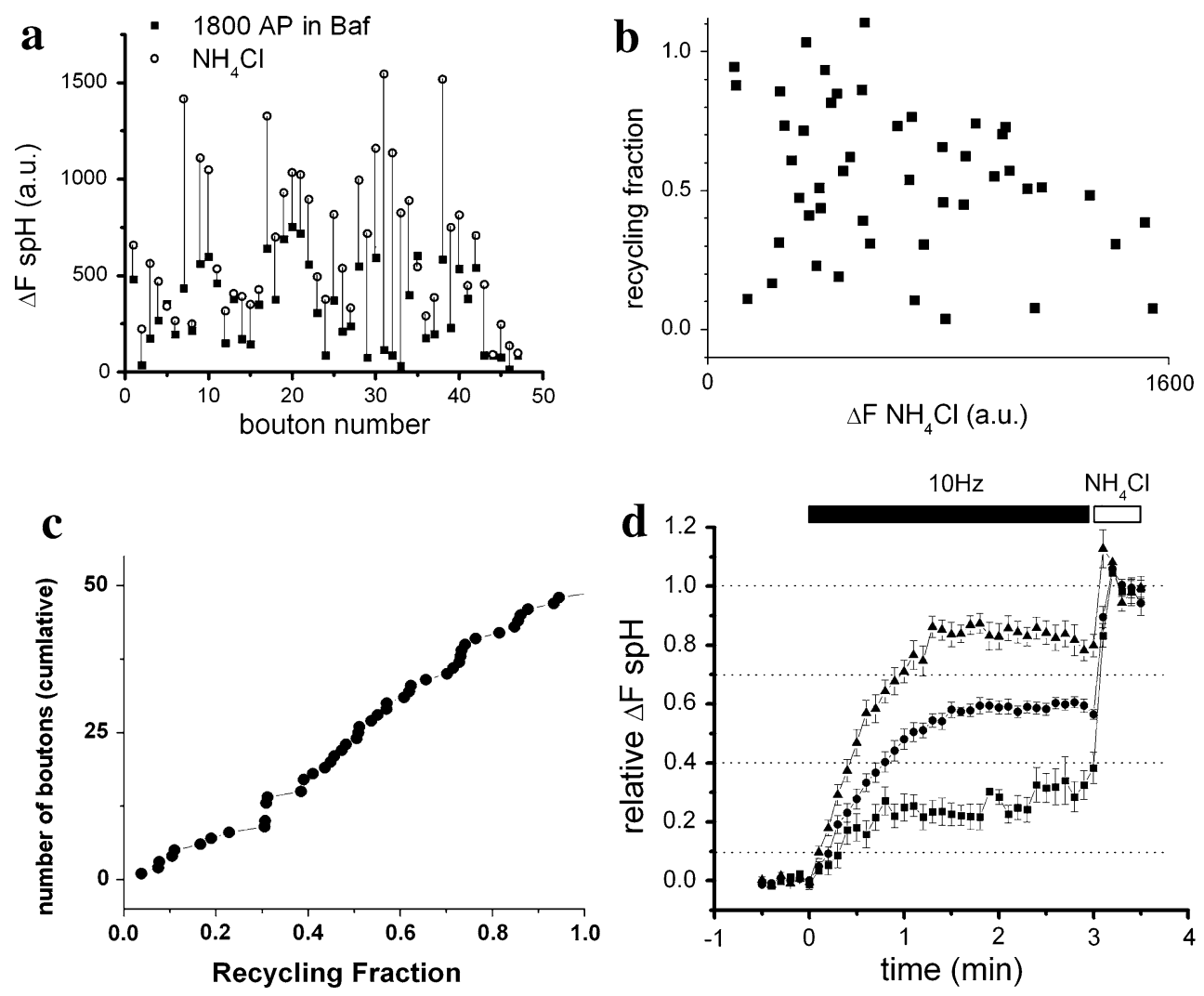

Fig. 2. Individual bouton analysis of the recycling vesicle pool fraction. The study of 47 boutons corresponding to one neuron (same as that presented in Fig. 1a) is shown. (a) The increase in spH fluorescence (with respect to the baseline at rest, $\Delta F \mathrm{spH}$ ) after $1800 \mathrm{APs}$ in baf (solid squares) and after $\mathrm{NH}_{4} \mathrm{Cl}$ perfusion (open circles) is shown for each bouton. The length of the line linking the symbols represents the size of the resting pool of vesicles. $\Delta F_{1800 \mathrm{AP}} / \Delta F_{\mathrm{NH}_{4} \mathrm{Cl}}$ was calculated to get the fraction recycling in (b) and (c). (b) The fraction of vesicles recycling is plotted as a function of the total vesicle pool size as reflected in the fluorescence increase in the presence of $\mathrm{NH}_{4} \mathrm{Cl}$. (c) Cumulative frequency histogram of the fraction of the total pool of vesicles that participates in recycling during prolonged stimulation (fraction recycling). The fraction recycling varied from 0 to 1 with an average value of $0.54 \pm 0.04$. (d) The fraction recycling does not appear to be related to the efficiency of the fusion machinery. Boutons were pooled in three groups according to the fraction of vesicles recycling: 0.1-0.4 (solid squares, $n=11$ ); $0.4-0.7$ (solid circles, $n=17$ ); and 0.7-1 (solid triangles, $n=14$ ). The average normalized fluorescence for each group is plotted as a function of time (dotted line delimits the three groups). The kinetics of exocytosis during stimulation ( 3 min at $10 \mathrm{~Hz}$ in baf, gray bar) is similar in the three groups $\left(\tau_{0.1-0.4}=32 \mathrm{~s}, \tau_{0.4-0.7}=37 \mathrm{~s}, \tau_{0.7-1}=32 \mathrm{~s}\right)$.

There were no obvious differences in the kinetics of vesicle pool mobilization and fusion among boutons that made use of a small, a medium, or a large fraction of its total pool to release neurotransmitter (0.1-0.4, 0.4-0.7, and 0.7-1 fraction groups, respectively), suggesting that the fraction recycling is independent of the efficiency of the fusion machinery (Fig. 2d). We found the average value to be $50 \pm 3 \%$ across 18 cells (Fig. 3a, b). Using vGlut-pHluorin (vGpH) or synaptotagmin I-pHluorin (tagmin-pH) as reporters in identical experiments, we found that the average recycling pool size was very similar (Fig. 3a, 60.1 $\pm 9.9 \%, v G \mathrm{vH}, n=5$;
$51.0 \pm 10 \%$ tagmin-pH, $n=4$ cells). Analysis of recycling fractions recycling over many cells shows that in addition to varying across boutons the mean recycling fraction also varies from neuron to neuron (Fig. 3b). The average fraction recycling was $22 \%$ and $67 \%$ for the neurons presenting the smallest and the highest values, respectively (cells number 14 and 11, respectively, see Fig. 3b). Similar ranges were obtained for $\mathrm{vGpH}$ and tagmin-pH. We also assessed whether the fraction recycling varied with age of cultures. It was recently reported that the amount of FM 1-43 uptake is twofold higher in neuronal cultures of 15 days versus 12 days 
a
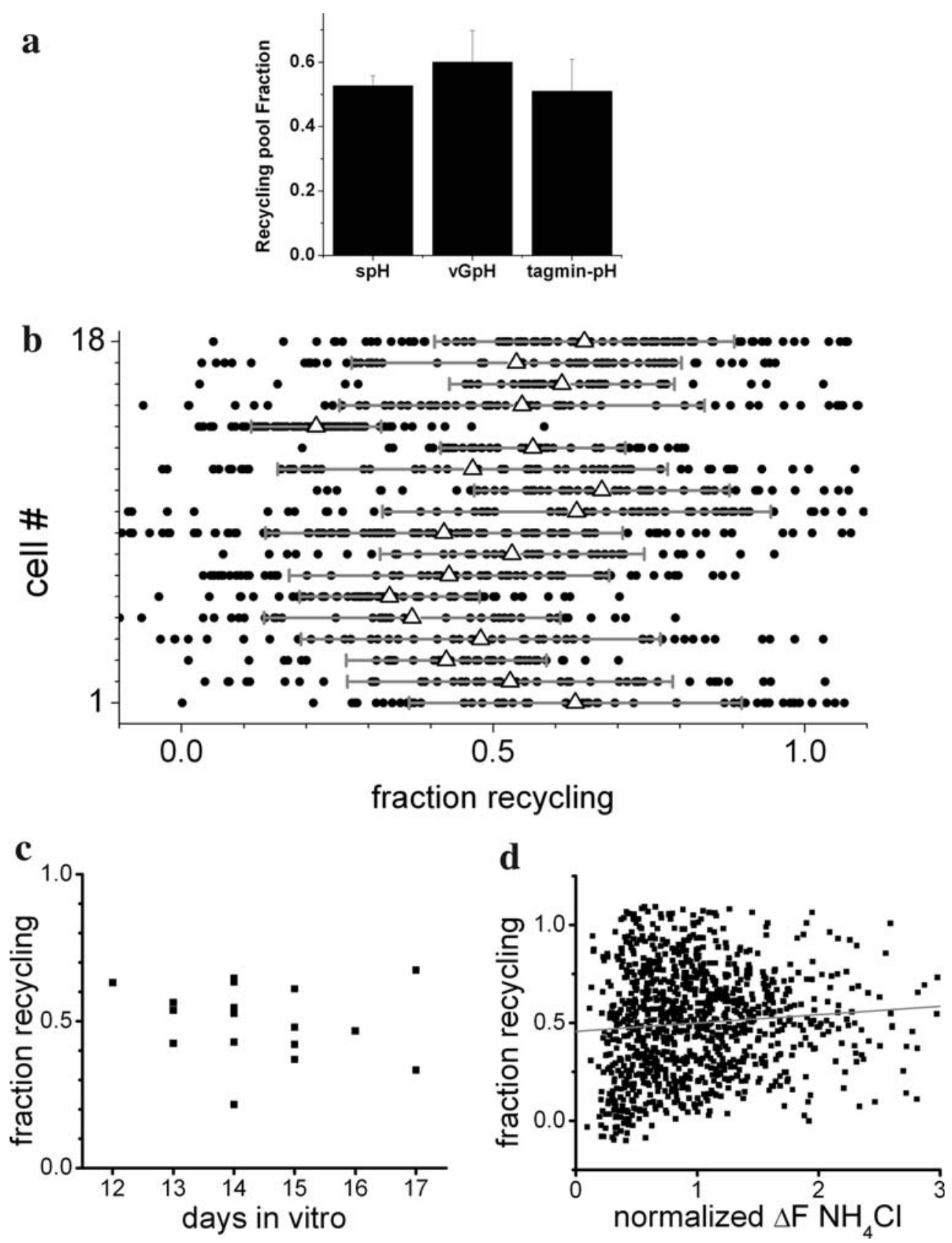

Fig. 3. The recycling vesicle fraction varies across different cells and synapses but represents on average $50 \%$ of the total pool. (a) Measurements of average fraction of recycling vesicles for $\mathrm{spH}(n=18)$, synaptotagmin-pHluorin (tagmin-pH, $n=4)$, and vGlut1-pHluorin $(\mathrm{vGpH}, n=5)$ are indistinguishable ( $P=0.05$ ANOVA). (b) Distribution of vesicle pool recycling fraction across different boutons in 18 cells from independent cultures. Each solid symbol represents a single measurement at a single bouton. The average recycling fraction and its standard deviation for each cell is shown as open triangles and gray bars, respectively $(n=36-96$ boutons in each cell). (c) The average fraction recycling for each experiment is plotted as a function of the age in vitro of the individual culture. The fraction recycling did not correlate with age in culture for the range of ages analyzed (12-17 days in vitro). (d) Boutons containing larger vesicle pools have a slight tendency to have higher recycling fractions. The increase in $\mathrm{spH}$ fluorescence in response to $\mathrm{NH}_{4} \mathrm{Cl}$ application $\left(\Delta F_{\mathrm{NH}_{4} \mathrm{Cl}}\right)$ gives an estimate of the size of the total vesicle pool. To compare across different neurons, this value was normalized to the average $\Delta F_{\mathrm{NH}_{4} \mathrm{Cl}}$ in each independent experiment. The gray line shows a linear fit to the data $(R=0.09 ; P<0.01)$. 
in vitro (Renger et al., 2001) suggesting that the number of vesicles recycling increases during synapse maturation. Contrary to what we expected, we found no increase in the fraction recycling in more mature cultures (Fig. 3c). However, it should be noted that if the total pool of vesicles increased proportionally to the recycling pool, then this fraction would not be affected. We also tested the hypothesis that boutons containing larger vesicle pools are able to engage a larger fraction of the pool in recycling. There is a very small $(R=0.09)$ but significant $(P<0.01)$ tendency of larger pools to recycle a larger fraction of its vesicles (Fig. $3 d$ ). Taken together, these experiments indicate that the fraction of the total pool of vesicles that recycles varies across synapses (0$100 \%)$ and across neurons ( $20-70 \%)$, that it is not correlated with the age of the culture and that terminals with more vesicles are likely to recycle a larger fraction of its vesicles.

\section{The fraction of vesicles recycling varies little in the time scale of hours}

The heterogeneity in the fraction of vesicles recycling across boutons from a single cell (Fig. 3) may indicate that, at the single bouton level, the fraction of vesicles recycling could be modulated over time. To test this hypothesis, we measured the change in the fraction recycling at single boutons over a period of $3 \mathrm{~h}$. Figure $4 \mathrm{a}$ shows that the average recycling fraction of all boutons remains stable over $3 \mathrm{~h}$. Interestingly, some boutons showed large variations in the fraction recycling (Fig. $4 \mathrm{~b}$, black bars). However, these represented only $19 \%$ of all boutons and included increasing and decreasing fraction of recycling vesicles (Fig. 4b). Thus, although a few boutons present large variation in the fraction recycling over time, the overall fraction across all boutons appeared to remain stable over a period of hours.

\section{Resting and recycling vesicles are interchanged across boutons}

The stability in the recycling and resting vesicle pools (Fig. 4) may indicate that vesicles remain stably bound at their terminals. Alternatively this may reflect a steady state behavior, with a constant flux of vesicles into and out of these pools, interchanging across boutons or even between recycling and resting pools within boutons while maintaining a constant relative abundance. To test these possibilities, we developed an approach to photobleach the fluorescence of the entire pool of vesicles (i.e., recycling and resting) in individual boutons and examined recovery of fluorescence over time of each of the pools (Fig. 5). We reasoned that photobleaching the $\mathrm{spH}$ fluorescence in the presence of $\mathrm{NH}_{4} \mathrm{Cl}$ at an individual bouton would irreversibly mark vesicles in both recycling and resting pools. A typical bleaching event is shown in Fig. 5. Prior to photobleaching the boutons show a robust $\mathrm{NH}_{4} \mathrm{Cl}$ response reflecting the alkalinization of both the recycling and resting vesicles. Following $\mathrm{NH}_{4} \mathrm{Cl}$ washout and a return to baseline fluorescence, the recycling pool can be mobilized to fuse with the plasma membrane with a $30 \mathrm{~s} 20 \mathrm{~Hz}$ stimulus (Fig. 5a). Individual boutons were then photobleached during a second $\mathrm{NH}_{4} \mathrm{Cl}$ challenge. Five minutes after this bleaching episode, the $\mathrm{NH}_{4} \mathrm{Cl}$ response at the bleached bouton was eliminated indicating both recycling and resting pools were bleached. A second round of AP stimulation gave rise to only a minimal signal, likely corresponding to diffusional spillover from adjacent nonbleached boutons (Sankaranarayanan and Ryan, 2000; Li and Murthy, 2001; Fernandez-Alfonso et al., 2006; Wienisch and Klingauf, 2006). Nearby boutons in the field retained both their $\mathrm{NH}_{4} \mathrm{Cl}$ signal as well as the stimulus-driven recycling pool signal. Repeated querying of fluorescence with $\mathrm{NH}_{4} \mathrm{Cl}$ and $\mathrm{AP}$ firing at different times after bleaching revealed that the fluorescence of both pools gradually returned to original values reaching near-completion within $\sim 3 \mathrm{~h}(87 \pm 7 \%$ of total pool, and $81 \pm 11 \%$ recycling fluorescence recovered, $n=3$ boutons) (Fig. 5b-d). A final stimulation in the presence of bafilomycin followed by $\mathrm{NH}_{4} \mathrm{Cl}$ confirmed that both recycling and resting vesicles are likely under dynamic exchange with neighboring boutons (Fig. 5e).

\section{Discussion}

We used a novel strategy to determine what fraction of vesicle pools in living synaptic terminals participates in vesicle recycling during prolonged stimulation. We relied upon measurements of genetically encoded tracers of synaptic vesicles, pHluorin-tagged synaptic vesicle proteins, to determine what portion of labeled vesicle pools can engage in recycling. In resting terminals, $\mathrm{spH}$ 
a
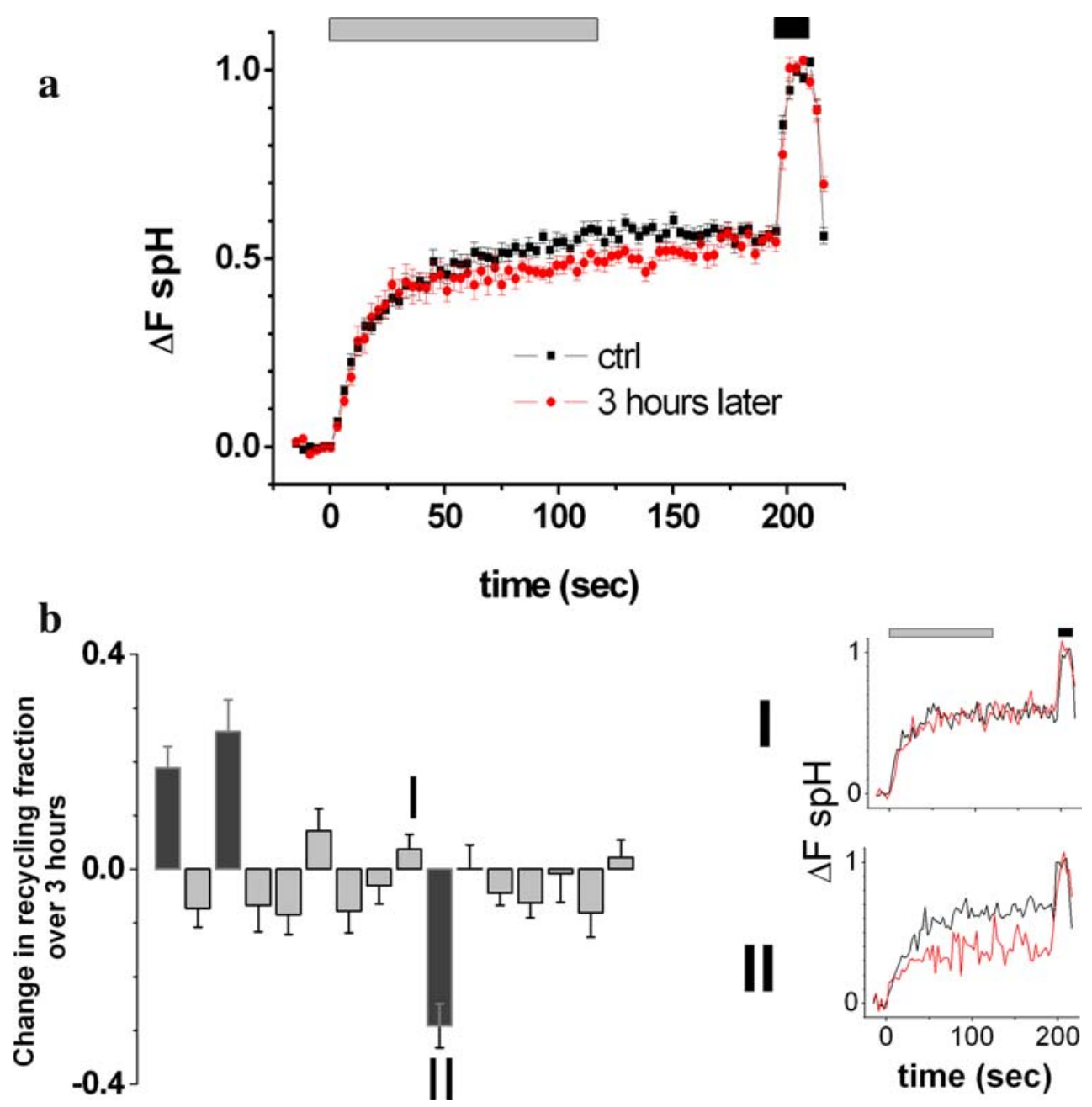

Fig. 4. The fraction of vesicles recycling is stable over a 3-h time period. (a) The average fraction of vesicles recycling measured in two consecutive trials with an interval of $3 \mathrm{~h}$ indicates that recycling and resting vesicles proportions are stable over time. Recycling fractions are $53 \pm 2 \%$ and $47 \pm 2 \%$ in the first and second trial, respectively ( $n=16$ boutons). (b) The majority of boutons showed little variation $(<10 \%)$ in the recycling fraction (13 boutons, dark gray bars). A few presented a larger variation (between $15 \%$ and $30 \%, n=3$ boutons, light gray bars). The error bars are the standard deviation of the steady-state fluorescence after all recycling vesicles were trapped in the alkaline state during the stimulus (2-6\%). The inset depicts the example of individual boutons presenting a $4 \%$ increase (I) and $29 \%$ decrease (II) in their recycling fraction.

fluorescence is quenched at the low vesicular $\mathrm{pH}$ $(\sim 5.5)$. During recycling in the presence of bafilomycin, a blocker of the vesicular proton pump responsible for vesicle reacidification, vesicles become trapped in the alkaline $(\mathrm{pH} \mathrm{7.4)}$ and thus fluorescent state. If all labeled vesicles in the pool recycle, then the fluorescence should reach a value that would be equivalent to direct alkalization of vesicles to $\mathrm{pH} 7.4$ (via either $\mathrm{NH}_{4} \mathrm{Cl}$ or protonophores). Any pHluorin-tagged protein that fails to recycle will remain acidic and maintain a low fluorescence value.

The robustness of our estimates of synaptic vesicle pool recycling fractions with pHluorintagged proteins relies on two main assumptions. First that the proteins we examined, VAMP-2, vGlut-1, and Synaptotagmin I, and their pHluorintagged variants, reside exclusively in synaptic vesicles at synaptic terminals. An alternate possibility is that some of the reporters may be targeted 
Fig. 5. Recycling and resting vesicle pools interchange across boutons. After assessing the size of the total vesicle pool with $\mathrm{NH}_{4} \mathrm{Cl}$ and estimating its recycling pool with a train of stimulation (a), all vesicles in a single bouton where bleached (red arrow in right panel pictures, red trace in left plots). (b-d) The same protocol applied 5 min (b), 30 min (c), and $160 \mathrm{~min}$ (d) after bleaching indicates that nonbleached vesicles reached the originally bleached bouton and distribute in both recycling and resting pools. (e) After $3 \mathrm{~h}$, nonbleached vesicles account for $91 \%$ of originally bleached ones $\left(\mathrm{NH}_{4} \mathrm{Cl}\right.$, compare with a); of these, $51 \%$ are capable of recycling (stimuli in Baf). The response to the same series of trials is also shown for a neighboring, nonbleached, bouton (a-e, white arrow in right panel pictures, black traces in left plots). Scale bar $5 \mu \mathrm{m}$.
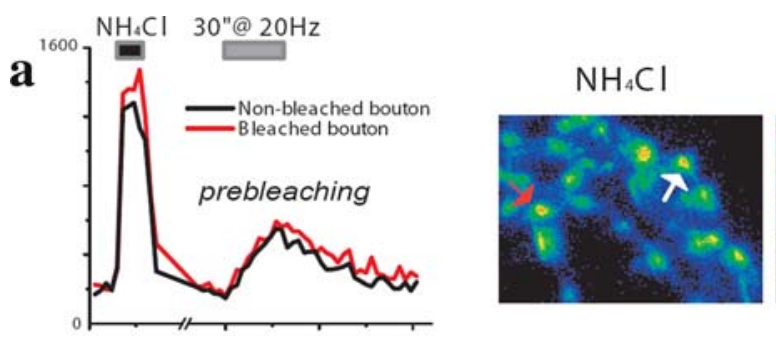

30"@20Hz
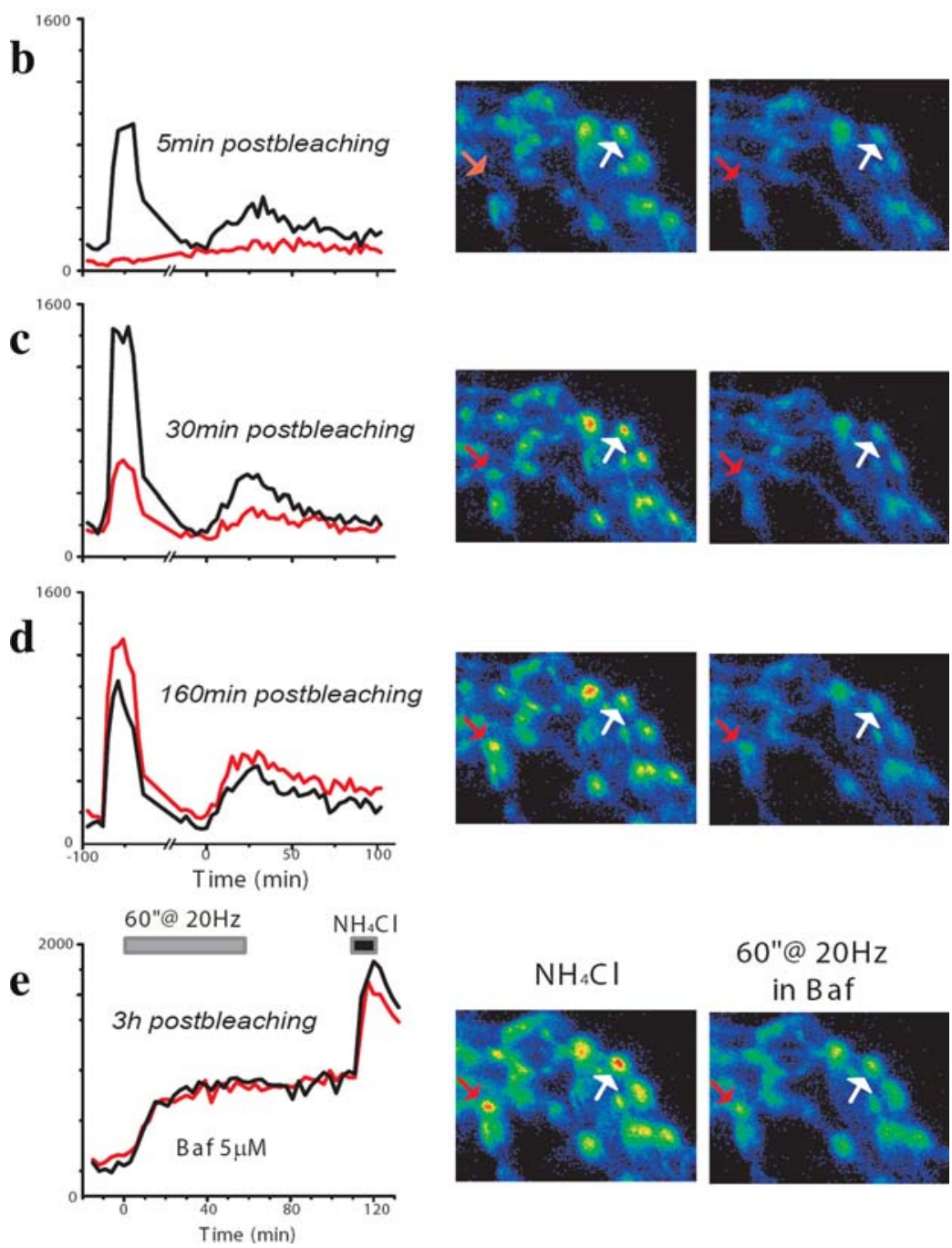

$\mathrm{NH}_{4} \mathrm{Cl}$

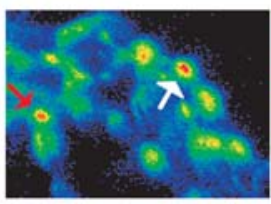

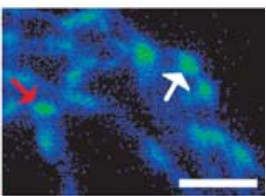

\section{.}


However, even if the expression of pHluorin-tagged SV proteins is biased toward one pool or another, the basic observation that a large fraction of the vesicles seem to be held in a state of "deep" reserve or are simply not competent for recycling will not change. We recently demonstrated that the expression of both spH (Fernandez-Alfonso et al., 2006) and $\mathrm{vGpH}$ (Balaji and Ryan, 2007) results in only 1-2 copies of the pHluorin-tagged protein per vesicle, much lower than the number of endogenous proteins likely to be present (Takamori et al., 2006). Thus, it seems unlikely that the appearance of these reporters in a resting compartment results from miss-targeting. Similarly, the fact that the most abundant synaptic vesicle proteins display similar partitioning argues that this is likely a bona-fide property of synaptic vesicles. Since we are measuring recycling vesicles relative to the total pool, it should be noted that, for example, a "silent" bouton does not necessarily mean a low fraction of vesicles recycling (i.e., a bouton that has a few vesicles recycling, although silent, will have a large fraction recycling if the total pool number is also very small; see Fig. 2a).

Our results are in reasonable agreement with other approaches for examining recycling vesicles based on photoconversion of FM 1-43 and electron microscopy (Harata et al., 2001). However, FM photoconversion experiments do not distinguish whether nonstained vesicles are the ones that fail to recycle or recycle in a manner that excludes dye labeling (i.e., kiss and run fusion). Since our method relies only on the exchange of protons within vesicles with the extracellular medium to track exocytosis, we conclude that resting vesicles are indeed the ones that do not fuse with the plasma membrane. Our approach also allows analyzing synaptic vesicle recycling in a vast number of synapses simultaneously, in living specimens, and in real time. We believe that the methods introduced in this work should add substantially in determining how various modulators of presynaptic function regulate recycling pool sizes.

Our photobleaching experiments indicate that both recycling and resting vesicle pools appear to exchange between synaptic boutons. Even at intermediate time points in the photobleaching recovery, the relative size of the recycling and resting pools (as judged by the peak of the $\mathrm{NH}_{4} \mathrm{Cl}$ compared to the peak of the stimulus response) appears constant. This suggests that the two pools are always closely linked, perhaps even constantly interchanging within a given synapse over these time scales. Recently, it was demonstrated that FM 1-43-labeled and thus by definition recycling vesicles, exchange between boutons (Darcy et al., 2006). Our results complement those studies and demonstrate that this sharing of vesicles extends to nonrecycling vesicles as well. Here our experiments do not distinguish between possible recovery by local interchange with a recycling pool (which itself can recover by surface diffusional exchange of $\mathrm{spH}$ or the slower intracellular exchange) or long range mobility of resting vesicles. It is interesting to point out that the time scale of recovery we see is considerably slower than the time scale of movement of recycling vesicle between boutons observed by Darcy et al. (2006).

In Drosophila neuromuscular junctions, two spatially segregated pools of vesicles have been characterized; one located in the periphery and the other in the central region of presynaptic terminals (Kuromi and Kidokoro, 1998). Interestingly, the central one was shown to participate in recycling only after high-frequency stimulation or activation of protein kinase A (Kuromi and Kidokoro, 2000). Results using an approach very similar to ours with pHluorin and alkaline trapping has also been used in drosophila, which confirmed the existence of a synaptic vesicle pool that could only successfully be mobilized following endocytic block in the shibire mutant at nonpermissive temperatures (Poskanzer and Davis, 2004), which had previously been observed using other methods (Delgado et al., 2000). It seems that there exists a pool of vesicles in a "deep reserve" state that can be switched to participate in recycling and is conserved across species for regulating synaptic transmission. It is important to note, however, that we did not find any obvious spatial distinction of the two pools in our experiments (compare panels ii and iii in Fig. 1a). Although it is true that our system does not provide high enough resolution to detect fine spatial distributions, electron micrographs of FM photoconversion experiments suggest that recycling and resting vesicles are distributed at random in mammalian CNS (Harata et al., 2001).

In conclusion, we show that a varying fraction of synaptic vesicles remain in a resting state (i.e., do not participate in recycling) during prolonged stimulation at CNS synapses. Although in a resting state, it is tempting to consider that part of this pool could be tapped to undergo recycling, that would imply that synapses have a large reserve capacity 
that might be used to modulate presynaptic function. It is also tempting to speculate that this resting pool might not only serve to regulate synaptic efficacy in individual boutons, but also be exchanged between synapses under some conditions. At present we do not know what physiological signal transduction pathways might normally control the balance of recycling and resting pools; however, the methods used here should prove useful in exploring how and when this "untapped" synaptic resource is utilized.

\section{Methods}

\section{Cell culture and experimental conditions} Hippocampal CA3-CA1 regions were dissected from 2- to 4-day-old Sprague-Dawley rats, dissociated, and plated onto poly-ornithine-coated glass as previously described (Ryan, 1999). Cultures were maintained at $37^{\circ} \mathrm{C}$ in a $95 \%$ air, $5 \% \mathrm{CO}_{2}$ humidified incubator, and culture media was replaced every week. In spH experiments, calcium phosphate-mediated gene transfer was used to transfect 6- to 8-day-old cultures as previously described (Sankaranarayanan et al., 2000) with super-ecliptic spH, vGpH, or Tagim-pH. Superecliptic pHluorin is a modified version of the original ecliptic pHluorin with enhanced optical properties but an identical $p K_{a}(\sim 7.1)$ for protonation (Sankaranarayanan et al., 2000). Cells were used 12 17 days after plating (6-12 days after transfection), and the coverslips were mounted in a rapid switching, laminar-flow perfusion and stimulation chamber on the stage of a custom-built laser scanning confocal microscope. The total volume of the chamber was $\sim 75 \mu \mathrm{l}$ and was perfused at a rate of $1-1.5 \mathrm{ml} / \mathrm{min}$. Action potentials were evoked by passing $1 \mathrm{~ms}$ current pulses, yielding fields of $\sim 10 \mathrm{~V} / \mathrm{cm}$ through the chamber via Platinum-Iridium electrodes. Unless otherwise noted cells were continuously perfused at room temperature $\left(\sim 24^{\circ} \mathrm{C}\right)$ in a saline solution containing in $\mathrm{mM}: 119$ $\mathrm{NaCl}, 2.5 \mathrm{KCl}, 2 \mathrm{CaCl}_{2}, 2 \mathrm{MgCl}_{2}, 25$ HEPES (buffered to $\mathrm{pH} 7.4$ ), 30 glucose, $10 \mu \mathrm{M}$ 6-cyano-7nitroquinoxaline-2,3-dione (CNQX; Research Biochemicals, Natick, MA) and $50 \mu \mathrm{M}$ D,L-2-amino-5-phosphonovaleric acid (AP5; Research Biochemicals). When external $\mathrm{CaCl}_{2}$ was varied, the total divalent ion concentration was kept constant by simultaneously changing $\mathrm{MgCl}_{2} . \mathrm{NaCl}$ and
$\mathrm{CaCl}_{2}$ were replaced with $\mathrm{KCl}$ (keeping the total ion concentration fixed) during application of Monensin (40 $\mu \mathrm{M}$, CalBiochem) and Nigericin (10 $\mu \mathrm{M}$, Molecular Probes), two carboxylic ionophores that together allow the exchange of cations for protons across cell membranes (both drugs were stored frozen as $10 \mathrm{mM}$ stock solutions in $\mathrm{MeOH})$. When $\mathrm{NH}_{4} \mathrm{Cl}(50 \mathrm{mM})$ was added to the saline, $\mathrm{NaCl}$ was reduced to $70 \mathrm{mM}$. Bafilomycin A1 (Baf; CalBiochem, San Diego, CA) was stored frozen as $0.2 \mathrm{mM}$ aliquots (in DMSO) and diluted to a final concentration of $0.5 \mu \mathrm{M}$ prior to $\mathrm{spH}$ experiments. In Fig. 1b, FM 4-64 (Molecular Probes, Eugene, OR) were used at a final concentration of $15 \mu \mathrm{M}$ and vesicles were labeled by electrical field stimulation for $30 \mathrm{~s}$ at $20 \mathrm{~Hz}$ in the presence of the dye in normal saline, followed by an additional $60 \mathrm{~s}$ of dye exposure to ensure labeling of all recycling membrane during endocytosis. Unless otherwise noted all chemicals were obtained from Sigma (St. Louis, MO).

\section{Optical measurements, microscopy, and analysis}

Laser-scanning fluorescence images were acquired using a custom-built laser-scanning microscope through a $40 \times 1.3$ numerical aperture Zeiss Fluar objective (Oberkochen, Germany). Specimens were illuminated with $\sim 1.5 \mu \mathrm{W}$ of the $488 \mathrm{~nm}$ line of an argon ion laser that was rapidly shuttered during all nondata acquiring periods using acoustooptic or electro-optic modulation. Time course of fluorescence response of $\mathrm{spH}$ and $\mathrm{FM}$ dyes was measured from time-lapse images taken every 3$10 \mathrm{~s}$. $\mathrm{SpH}$ fluorescence emission was collected using a $500 \mathrm{~nm}$ long pass filter (except for combined FM 4-64 and spH experiments, where a 498$538 \mathrm{~nm}$ band pass filter was used for $\mathrm{spH}$ ); FM 464 emission was collected using a $650 \mathrm{~nm}$ long pass filter. Quantitative measurements of fluorescence intensity at individual boutons were obtained by averaging $4 \times 4$ area pixels $\left(0.4 \times 0.4 \mu \mathrm{m}^{2}\right)$ corresponding to the center of mass of $8 \times 8$-pixels positioned over individual puncta selected by hand from images (Fig. 1a shows a subset of areas selected). Further criteria for including a fluorescent puncta as a synaptic bouton in our analysis included requiring a minimum of a 1.5-fold increase in fluorescence upon alkalization with $\mathrm{NH}_{4} \mathrm{Cl}$ compared to that at rest $\left(\left[F_{\mathrm{NH}_{4} \mathrm{Cl}}-F_{\text {rest }}\right] / F_{\text {rest }}\right)$. Fluorescence values for baseline, prolonged stimulation 
and $\mathrm{NH}_{4} \mathrm{Cl}$ were obtained by averaging $2-5$ images at rest, after reaching a steady-state level during stimulation (10 or $20 \mathrm{~Hz}$ ), and during $\mathrm{NH}_{4} \mathrm{Cl}$ exposure, respectively. The noise in our estimates of fraction recycling was calculated from fluorescence difference of the average of the first three images and the average of the following three images taken prior to stimulation (six images were taken before stimulation), relative to the average change in fluorescence in $\mathrm{NH}_{4} \mathrm{Cl}$ (i.e., $\left[F_{1-3}-F_{4-6}\right] /$ $\left.\Delta F_{\mathrm{NH}_{4} \mathrm{Cl}}\right)$. The standard deviation of the estimated noise was 0.11 ( $n=148$ boutons), therefore the fraction recycling was binned in 0.1 intervals. Four out of 22 neurons, which had high basal rates of fluorescence increase on bafilomycin application, were not included in the final analysis, since we could not distinguish with confidence whether the rise in fluorescence was due to spontaneous alkalization of vesicles or spontaneous vesicle fusions (Sankaranarayanan and Ryan, 2001). Data points that presented a fraction recycling of less than -0.1 or more than 1.1 probably represented slight shifts of boutons from focal plane and were not included in our analysis (55 out of 1,085 boutons). $\Delta F$ for each bouton was always normalized to $\Delta F_{\mathrm{NH}_{4} \mathrm{Cl}}$ before any further analysis. In Fig. 5, bleaching was achieved by scanning the laser beam over a region of interest encompassing a single bouton ( 10 times more power and scanning rates than for regular imaging) in the presence of $\mathrm{NH}_{4} \mathrm{Cl}$ for $\sim 30 \mathrm{~s}$. Errors are shown as standard error of mean except where stated.

\section{Acknowledgments}

We would like to thank Dr. James Rothman for kindly providing the super-ecliptic synapto-pHluorin and tagmin-pHluorin constructs, Robert Edwards and Susan Voglemeier for the vGlut-1-pHluorin construct, members of the Ryan lab for useful discussions, and Wayne Yan and Ricky Kwan for excellent technical assistance. Data using vGlutpHluorin and tagmin-pHluorin were kindly provided by Sung Yung Kim and Pablo Ariel. Sethu Sankaranarayanan kindly provided some of the data using $\mathrm{spH}$. This work was supported by $\mathrm{NIH}$ (NS24992) a McKnight Technological Innovations in Neuroscience Award and an Irma T. Hirshcl career scientist award to T.A.R.

\section{Open Access}

This article is distributed under the terms of the Creative Commons Attribution Noncommercial License which permits any noncommercial use, distribution, and reproduction in any medium, provided the original author(s) and source are credited.

\section{References}

Balaji, J. and Ryan, T. A. (2007). Single-vesicle imaging reveals that synaptic vesicle exocytosis and endocytosis are coupled by a single stochastic mode. Proc. Natl Acad. Sci. USA 104, 20576-20581.

Betz, W. J. and Angleson, J. K. (1998). The synaptic vesicle cycle. Annu. Rev. Physiol. 60, 347-363.

Cousin, M. A. and Nicholls, D. G. (1997). Synaptic vesicle recycling in cultured cerebellar granule cells: role of vesicular acidification and refilling. J. Neurochem. 69, 1927-1935.

Darcy, K. J., Staras, K., Collinson, L. M., and Goda, Y. (2006). Constitutive sharing of recycling synaptic vesicles between presynaptic boutons. Nat. Neurosci. 9, 315-321.

de Lange, R. P., de Roos, A. D., and Borst, J. G. (2003). Two modes of vesicle recycling in the rat calyx of Held. J. Neurosci. 23, 10164-10173.

Delgado, R., Maureira, C., Oliva, C., Kidokoro, Y., and Labarca, P. (2000). Size of vesicle pools, rates of mobilization, and recycling at neuromuscular synapses of a Drosophila mutant, shibire. Neuron 28, 941-953.

Dobrunz, L. E. and Stevens, C. F. (1997). Heterogeneity of release probability, facilitation, and depletion at central synapses. Neuron 18, 9951008.

Elmqvist, D. and Quastel, D. M. (1965). A quantitative study of end-plate potentials in isolated human muscle. J. Physiol. 178, 505-529.

Fernandez-Alfonso, T., Kwan, R., and Ryan, T. A. (2006). Synaptic vesicles interchange their membrane proteins with a large surface reservoir during recycling. Neuron 51, 179-186.

Fernandez-Alfonso, T. and Ryan, T. A. (2006). The efficiency of the synaptic vesicle cycle at central nervous system synapses. Trends Cell Biol. 16, 413-420. 
Granseth, B., Odermatt, B., Royle, S. J., and Lagnado, L. (2006). Clathrin-mediated endocytosis is the dominant mechanism of vesicle retrieval at hippocampal synapses. Neuron 51, 773-786.

Harata, N., Ryan, T. A., Smith, S. J., Buchanan, J., and Tsien, R. W. (2001). Visualizing recycling synaptic vesicles in hippocampal neurons by FM 1-43 photoconversion. Proc. Natl. Acad. Sci. USA 98, 12748-12753.

Kuromi, H. and Kidokoro, Y. (1998). Two distinct pools of synaptic vesicles in single presynaptic boutons in a temperature-sensitive Drosophila mutant, shibire. Neuron 20, 917-925.

Kuromi, H. and Kidokoro, Y. (2000). Tetanic stimulation recruits vesicles from reserve pool via a cAMP-mediated process in Drosophila synapses. Neuron 27, 133-143.

Lee, H. C. and Epel, D. (1983). Changes in intracellular acidic compartments in sea urchin eggs after activation. Dev. Biol. 98, 446-454.

Lemasters, J. J., Chacon, E., Ohata, H., Harper, I. S., Nieminen, A. L., Tesfai, S. A., and Herman, B. (1995). Measurement of electrical potential, $\mathrm{pH}$, and free calcium ion concentration in mitochondria of living cells by laser scanning confocal microscopy. Methods Enzymol. 260, 428-444.

Li, Z., Burrone, J., Tyler, W. J., Hartman, K. N., Albeanu, D. F., and Murthy, V. N. (2005). Synaptic vesicle recycling studied in transgenic mice expressing synaptopHluorin. Proc. Natl. Acad. Sci. USA 102, 6131-6136.

Li, Z. and Murthy, V. N. (2001). Visualizing postendocytic traffic of synaptic vesicles at hippocampal synapses. Neuron 31, 593-605.

Miesenbock, G., De Angelis, D. A., and Rothman, J. E. (1998). Visualizing secretion and synaptic transmission with $\mathrm{pH}$-sensitive green fluorescent proteins. Nature 394, 192-195.

Mukherjee, S., Ghosh, R. N., and Maxfield, F. R. (1997). Endocytosis. Physiol. Rev. 77, 759803.

Murthy, V. N., Sejnowski, T. J., and Stevens, C. F. (1997). Heterogeneous release properties of visualized individual hippocampal synapses. Neuron 18, 599-612.

Murthy, V. N. and Stevens, C. F. (1999). Reversal of synaptic vesicle docking at central synapses. Nat. Neurosci. 2, 503-507.

Neher, E. (1998). Vesicle pools and Ca2+ microdomains: new tools for understanding their roles in neurotransmitter release. Neuron 20, 389-399.

Pieribone, V. A., Shupliakov, O., Brodin, L., HilfikerRothenfluh, S., Czernik, A. J., and Greengard, P. (1995). Distinct pools of synaptic vesicles in neurotransmitter release. Nature 375, 493497.

Poskanzer, K. E. and Davis, G. W. (2004). Mobilization and fusion of a non-recycling pool of synaptic vesicles under conditions of endocytic blockade. Neuropharmacology 47, 714-723.

Renger, J. J., Egles, C., and Liu, G. (2001). A developmental switch in neurotransmitter flux enhances synaptic efficacy by affecting AMPA receptor activation. Neuron 29, 469-484.

Ryan, T. A. (1999). Inhibitors of myosin light chain kinase block synaptic vesicle pool mobilization during action potential firing. J. Neurosci. 19, 1317-1323.

Ryan, T. A., Li, L., Chin, L. S., Greengard, P., and Smith, S. J. (1996). Synaptic vesicle recycling in synapsin I knock-out mice. J. Cell Biol. 134, 1219-1227.

Ryan, T. A. and Smith, S. J. (1995). Vesicle pool mobilization during action potential firing at hippocampal synapses. Neuron 14, 983-989.

Sankaranarayanan, S., De Angelis, D., Rothman, J. E., and Ryan, T. A. (2000). The use of pHluorins for optical measurements of presynaptic activity. Biophys. J. 79, 2199-2208.

Sankaranarayanan, S. and Ryan, T. A. (2000). Real-time measurements of vesicle-SNARE recycling in synapses of the central nervous system. Nat. Cell Biol. 2, 197-204.

Sankaranarayanan, S. and Ryan, T. A. (2001). Calcium accelerates endocytosis of vSNAREs at hippocampal synapses. Nat. Neurosci. 4, 129-136.

Stevens, C. F. and Tsujimoto, T. (1995). Estimates for the pool size of releasable quanta at a single central synapse and for the time required to refill the pool. Proc. Natl. Acad. Sci. USA 92, 846-849.

Sudhof, T. C. (2000). The synaptic vesicle cycle revisited. Neuron 28, 317-320.

Takamori, S., Holt, M., Stenius, K., Lemke, E. A., Gronborg, M., Riedel, D., Urlaub, H., Schenck, S., Brugger, B., Ringler, P. et al. (2006). Molecular anatomy of a trafficking organelle. Cell 127, 831-846.

Voglmaier, S. M., Kam, K., Yang, H., Fortin, D. L., Hua, Z., Nicoll, R. A., and Edwards, R. H. 
(2006). Distinct endocytic pathways control the rate and extent of synaptic vesicle protein recycling. Neuron 51, 71-84.

von Gersdorff, H. and Matthews, G. (1997). Depletion and replenishment of vesicle pools at a ribbon-type synaptic terminal. J. Neurosci. 17, 1919-1927.

von Gersdorff, H. and Matthews, G. (1999). Electrophysiology of synaptic vesicle cycling. Annu. Rev. Physiol. 61, 725-752.

West, A. E., Neve, R. L., and Buckley, K. M. (1997). Targeting of the synaptic vesicle protein synaptobrevin in the axon of cultured hippocampal neurons: evidence for two distinct sorting steps. J. Cell Biol. 139, 917-927.
Wienisch, M. and Klingauf, J. (2006). Vesicular proteins exocytosed and subsequently retrieved by compensatory endocytosis are nonidentical. Nat. Neurosci. 9, 1019-1027.

Wu, L. G. and Betz, W. J. (1998). Kinetics of synaptic depression and vesicle recycling after tetanic stimulation of frog motor nerve terminals. Biophys. J. 74, 3003-3009.

Zhou, Q., Petersen, C. C., and Nicoll, R. A. (2000). Effects of reduced vesicular filling on synaptic transmission in rat hippocampal neurones. J. Physiol. 525 Pt 1, 195-206. 